\title{
DISJOINT SUM FORMS IN RELIABILITY THEORY
}

\author{
B. ANRIG ${ }^{1}$, F. BEICHELT ${ }^{2}$ \\ ${ }^{1}$ University of Fribourg, Department of Informatics, Rue de Faucigny 2, \\ 1700 Fribourg, Switzerland \\ ${ }^{2}$ University of the Witwatersrand, School of Statistics and Actuarial Science \\ Private Bag 3, WITS 2050, Johannesburg, South Africa
}

\begin{abstract}
The structure function $\varphi$ of a binary monotone system is assumed to be known and given in a disjunctive normal form, i.e. as the logical union of products of the indicator variables of the states of its subsystems. Based on this representation of $\varphi$, an improved Abraham algorithm is proposed for generating the disjoint sum form of $\varphi$. This form is the base for subsequent numerical reliability calculations. The approach is generalized to multivalued systems. Examples are discussed.
\end{abstract}

\section{INTRODUCTION}

We deal with a basic problem of reliability theory, namely with finding and analyzing algorithms for calculating reliability criteria of systems based on reliability criteria of its elements (subsystems). Even in the simple case of binary monotone systems with independent elements, these algorithms generally have running times which increase exponentially fast with the complexity of the system [5]. Within that limitation it is, however, imperative to develop algorithms, which are relatively fast and applicable to a broad class of problems. The first step is to determine the structure function of the system. This paper requires the structure function to be known and given in a disjunctive normal form.

\section{BINARY SYSTEMS}

Let $S$ be the system under consideration and $e_{1}, e_{2}, \ldots, e_{n}$ its $n$ elements. Let furthermore $z_{s}$ be indicator variable of the system state and $z_{i}$ the indicator variable of element $e_{i}$. Then, in a binary system, 


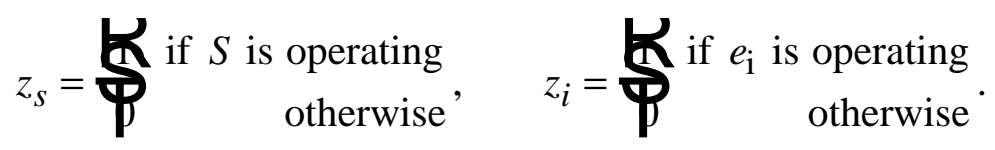

The structure function $\varphi$ of $S$ is a Boolean function, which describes the mutual relationship between the states of the system and its elements:

$$
z_{s}=\varphi\left(z_{1}, z_{2}, \ldots, z_{n}\right) .
$$

$z_{s}$ and the $z_{i}$ are Boolean, binary or, more specific, (0-1)-variable. For any two (0-1)variable $x$ and $y$, disjunction, conjunction and complement are defined as follows:

Disjunction $\quad x \vee y=x+y-x y=\max (x, y)$

Conjunction $\quad x \wedge y=x y=\min (x, y)$

Negation $\quad \bar{x}=1-x$

Furthermore, $x$ and $y$ are called disjoint if $x y=0$. Hence,

$$
x \vee y=x+y \quad \text { if } x \text { and } y \text { are disjoint . }
$$

Let $\mathbf{Z}$ be the set of all $2^{n}$ state vectors of the system. The system is monotone if $\varphi$ is nondecreasing in each variable $z_{i}$ and each $z_{i}$ is relevant with respect to $\varphi$, i.e. $z_{i}$ influences the value of $\varphi$. A vector $\vec{z} \in \mathbf{Z}$ is called a path vector of $\varphi$ if $\varphi(\vec{z})=1$. A path vector $\vec{z}$ is called minimal if $\varphi(\vec{y})=0$ for all $\vec{y} \in \mathbf{Z}$ with $\vec{y}<\vec{z}$. Note that $\vec{y}=\left(y_{1}, y_{2}, \ldots, y_{n}\right)<\vec{z}=\left(z_{1}, z_{2}, \ldots, z_{n}\right)$ iff $y_{i}<z_{i}$ for all $i=1,2, \ldots, n$ and $y_{j}<z_{j}$ for at least one $j$. Let $\vec{z}_{1}, \vec{z}_{2}, \ldots, \vec{z}_{w}$ be the set of all minimal path vectors. The minimal path set belonging to $\vec{z}=\left(z_{1}, z_{2}, \ldots, z_{n}\right)$ is defined as

$$
W_{i}=\text { П } z_{j}=1 \mathbf{c}
$$

Obviously, the concepts of (minimal) path vectors and (minimal) path sets are equivalent. For a thorough discussion of these concepts see, for instance, [7] and [15]. It is easy to see that the structure function of any binary monotone system with minimal path sets $W_{1}, W_{2}, \ldots, W_{w}$ has structure

$$
\varphi(\vec{z})=A_{1} \vee A_{2} \vee \cdots \vee A_{w}
$$

with

$$
A_{j}=\prod_{i \in W_{j}} z_{i} ; j=1,2, \ldots, w
$$


Since there are computer-aided methods for determining the minimal path sets of any binary monotone system, the disjunctive normal form of $\varphi$ given by (2) is most widely used for generating disjoint sum forms of structure functions. A disjoint sum form of $\varphi$ has structure

$$
\varphi(\vec{z})=D_{1}+D_{2}+\cdots+D_{d} ; \quad D_{i} D_{j}=0, i \neq j,
$$

where the $D_{k} ; k=1,2, \ldots, d$; are products of some $z_{i}$ and $\bar{z}_{j} ; i \neq j$. Hence, $\varphi$ given by (3) is a sum of mutually disjoint terms $D_{i}$.

Let us now take into account that the $z_{i}$ are random variables with

$$
z_{i}=\underset{\mathbf{W}}{\text { with probability } p_{i}} \begin{aligned}
& \text { with probability } \bar{p}_{i}=1-p_{i} \\
& ; i=1,2, \ldots, n ;
\end{aligned}
$$

and let $p_{s}=P\left(z_{s}=1\right)=P(\varphi(\vec{z})=1)$ be the probability that the system is operating. $p_{s}$ is called the availability of the system and $p_{i}$ is the availability of element $e_{i}$. Since $z_{s}=\varphi(\vec{z})$ is a binary random variable,

$$
p_{s}=E(\varphi(\vec{z})) .
$$

This relationship shows the practical importance of a disjoint sum form (3): The system availability is simply given by

$$
p_{s}=E\left(D_{1}\right)+E\left(D_{2}\right)+\cdots+E\left(D_{d}\right) .
$$

If the $z_{i}$ are independent random variables, i.e. the elements operate independently from each other, then $E\left(D_{i}\right)$ is obtained from $D_{i}$ simply by replacing there the $z_{i}$ and $\bar{z}_{j}$ with the corresponding $p_{i}$ and $\bar{p}_{j}$, respectively.

Structure functions, in particular disjoint sum forms, are not unique. For the sake of computational efficiency, a disjoint sum form should be of low complexity, i.e. both $d$ and the total number of factors in the $D_{i}$ should be small. Numerous algorithms transforming the disjunctive normal form (2) into a disjoint sum form of low complexity have been developed. Most popular is the algorithm of Abraham [2]. It has formed the basis for substantially improved versions yielding disjoint sum forms of lower complexity than the original version of Abraham [7], [8], [9], [14]. The probably most efficient algorithm not based on Abraham's one, is due to Torrey [22]. For surveys, see [21], [23]. 
The following version of Abraham's algorithm is based on an algorithm given in [7]. To describe the algorithm, some further notation is needed. Let $M$ be the set of all possible products of some $z_{i}$ and $\bar{z}_{j}$. The principle of the algorithm consists in replacing each $A_{k}$ in (2) with a sum of disjoint products

$$
L_{k}=\sum_{D \in M_{k}} D, \quad M_{k} \subseteq M
$$

such that

$$
A_{1} \vee A_{2} \vee \cdots \vee A_{w}=\sum_{k=1}^{w} L_{k}=\sum_{D \in M_{\varphi}} D
$$

with $L_{1}=A_{1}$ and the set $M_{\varphi}=M_{1} \cup M_{2} \cup \cdots M_{w}$ consists of mutual disjoint products from $M$. Thus, the set $M_{\varphi}$ can be identified with the structure function $\varphi$. The sums $L_{k}$ are successively generated from sums $L_{1, k}, L_{2, k}, \cdots, L_{k-1, k}$ with property

$$
A_{1} \vee A_{2} \vee \cdots \vee A_{j} \vee A_{k}=A_{1} \vee A_{2} \vee \cdots \vee A_{j}+L_{j, k}
$$

where

$$
L_{j, k}=\sum_{D \in M_{j, k}} D, \quad M_{j, k} \subseteq M
$$

The process starts for each $k=2,3, \ldots, w$ at $j=1$ and stops at $j=k-1, L_{k-1, k}=L_{k}$. The transition from $L_{j-1, k}$ to $L_{j, k}$ or, equivalently, from $M_{j-1, k}$ to $M_{j, k}$, depends on which of the following three cases occurs. To characterize these cases, let $A$ be the product of some $z_{i}$ and $C(A, B)=\left.\right|_{C_{1}}, C_{2}, \ldots, C_{c} \mathbf{Q} B \in M$, the set of all those $z_{i}$, which are factors in $A$, but not in $B$.

a) $\quad A \cdot B=\varnothing$ ( $A$ and $B$ are disjoint) if $z_{i}$ is a factor in $A$ and $\bar{z}_{i}$ is a factor in $B$.

b) $A \vee B=A$ if $A$ and $B$ are not disjoint and $C(A, B)=\varnothing$.

c) $A \vee B=A+\bar{C}_{1} B+C_{1} \bar{C}_{2} B+\cdots+C_{1} C_{2} \cdots C_{c-1} \bar{C}_{c} B$ if $A$ and $B$ are not disjoint and $C(A, B) \neq \varnothing$.

To construct $M_{j, k}$ from $M_{j-1, k}, C\left(A_{j}, B\right)$ is determined for $B \in M_{j-1, k}$. If case a) applies, then $B$ also is an element of $M_{j, k}$. If $\left.\mathrm{b}\right)$ is true, then $B$ is eliminated, since it does not contribute to the construction of $M_{k-1, k}=M_{k}$. In case c), $M_{j, k}$ contains the products $\bar{C}_{1} B, C_{1} \bar{C}_{2} B, \cdots, C_{1} C_{2} \cdots C_{c-1} \bar{C}_{c} B$. The complete set $M_{j, k}$ is obtained if this procedure is 
repeated for all $B \in M_{j-1, k}$ and starts with $M_{0, k}=\left.\right|_{A_{k}} \mathbf{C}$. Note that the sets $M_{k}$ are generated independently from each other. Hence, they can be determined in an arbitrary order.

\section{Algorithm 1}

1 Order the $A_{j}$ according to the number of their factors.

$2 \quad$ Initialize $M_{\varphi}=\mathbf{I} A_{1} \mathbf{Q} k=2$

$3 \quad$ Initialize $M_{0, k}=\mid A_{k} \mathbf{Q} k=2$

$4 \quad$ Initialize $M_{j, k}=\varnothing$

5 For all $B \in M_{j-1, k}$ :

5.1 If $A_{j}$ and $B$ are disjoint, $B$ becomes element of $M_{j, k}$. Select another $B$.

5.2 Determine $C\left(A_{j}, B\right)$.

5.3 If $C\left(A_{j}, B\right)=\varnothing$, delete $B$ and select another $B$.

5.4 If $C\left(A_{j}, B\right)=\mid C_{1}, C_{2}, \ldots, C_{c} \mathbf{Q} c \geq 1$, then $\bar{C}_{1} B, C_{1} \bar{C}_{2} B, \cdots, C_{1} C_{2} \cdots C_{c-1} \bar{C}_{c} B$ become elements of $M_{j, k}$.

5.5 Select another $B$.

6 If $j<k-1$, then $j \leftarrow j+1$ and go to 4 .

7 Expand $M_{\varphi}$ by adding $M_{k-1, k}$.

8 If $k<w$, then $k \leftarrow k+1$ and go to 3. If $k=w$, STOP.

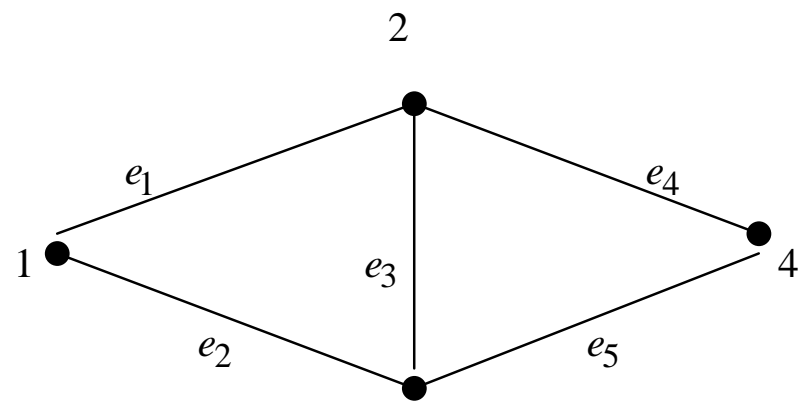

3

Figure 1 Bridge structure

Example 1 Let us consider a system the reliability block diagram of which is given by the "bridge structure" (Figure 1), i.e. it has the four nodes 1, 2, 3 and 4 with 1 and 4 being 
entrance and exit nodes, respectively, and edges $e_{1}=(1,2), e_{2}=(1,3), e_{3}=(2,3), e_{4}=(2,4)$, and $e_{5}=(3,4)$.

From Figure $1, W_{1}=\mathbf{|}, 4 \mathbf{C}, W_{2}=\mathbf{|} 2,5 \mathbf{C}, W_{3}=\mathbf{l}, 3,5 \mathbf{C}, W_{4}=\mathbf{I}_{2,3,4} \mathbf{C}$. Hence,

$$
A_{1}=z_{1} z_{4}, A_{2}=z_{2} z_{5}, A_{3}=z_{1} z_{3} z_{6}, A_{4}=z_{2} z_{3} z_{4},
$$

so that the disjunctive normal form (2) is given by

$$
\varphi(\vec{z})=z_{1} z_{4} \vee z_{2} z_{5} \vee z_{1} z_{3} z_{5} \vee z_{2} z_{3} z_{4} .
$$

To apply algorithm 1, firstly, initialize $M_{0,2}=\mathbf{I}_{2}$ C. Then $B=A_{2} \in M_{0,2}$ is selected. Since

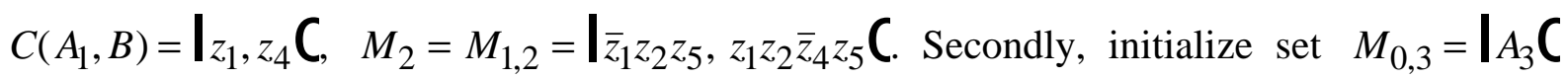

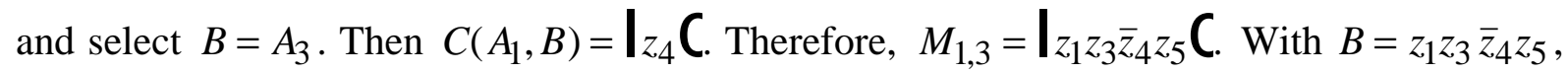
$C\left(A_{2}, B\right)=\left.\right|_{z_{2}} \mathbf{C}$. Thus, $M_{3}=M_{2,3}=\left.\right|_{z_{1}} \bar{z}_{2} z_{3} \bar{z}_{4} z_{5} \mathbf{C}$. Thirdly, initialize $M_{0,4}=\left.\right|_{A_{4}} \mathbf{C}$. Then, with $B=A_{4}, \quad C\left(A_{1}, B\right)=\left.\right|_{z_{1}} \mathbf{C}$. This gives $M_{1,4}=\left.\right|_{\overline{z_{1}}} z_{2} z_{3} z_{4} \mathbf{C}$. With $B=\bar{z}_{1} z_{2} z_{3} z_{4}$, the corresponding set $C\left(A_{2}, B\right)$ becomes $C\left(A_{2}, B\right)=\mid z_{5} \mathbf{C}$. Therefore, $M_{2,4}=\mid \bar{z}_{1} z_{2} z_{3} z_{4} \bar{z}_{5} \mathbf{C}$. Lastly, with $B=\bar{z}_{1} z_{2} z_{3} z_{4} \bar{z}_{5}, A_{3}$ and $B$ are disjoint (case a). Hence, $B$ is also element of $M_{3,4}$. This implies $M_{4}=M_{3,4}=M_{2,4}$. In view of $M_{\varphi}=M_{1} \cup M_{2} \cup M_{3} \cup M_{4}$ with $M_{1}=A_{1}$, the disjoint sum form is

$$
\varphi(\vec{z})=z_{1} z_{4}+\bar{z}_{1} z_{2} z_{5}+z_{1} z_{2} \bar{z}_{4} z_{5}+z_{1} \bar{z}_{2} z_{3} \bar{z}_{4} z_{5}+\bar{z}_{1} z_{2} z_{3} z_{4} \bar{z}_{5} .
$$

Algorithm 1 is not applicable to generating disjoint sum forms of non-monotone structure functions. However, there are technical systems, whose reliability behaviour can only be described by non-monotone structure functions. Examples are given in [7]. Moreover, the problem of generating disjoint sum forms from disjunctive normal forms also arises in probabilistic model-based reasoning and in the Dempster-Shafer theory of evidence. Here the Boolean functions of interest ("structure functions") are usually non-monotone. Abraham's approach to generating disjoint sum forms from disjunctive normal forms of Boolean functions has been generalized to non-monotone Boolean and even multi-valued functions in $[3,4], 10,12,16,17,18,20]$.

\section{MULTIVALUED SYSTEMS}

To assume that the system $S$ and its elements $e_{1}, e_{2}, \ldots, e_{n}$ can only be in either state "available" or "not available" is frequently an inadmissible oversimplification of the real 
situation. Consider, for example, systems (elements) with different operating and/or failure modes. Hence, it makes sense to assume that the indicator variables $z_{s}$ and $z_{i}$ of the states of $S$ and $e_{i}$ can assume values from sets

$$
\mathbf{z}_{s}=\mathbf{Q}_{s, 1}, z_{s, 2}, \ldots, z_{s, r_{s}} \mathbf{t} \text { and } \mathbf{z}_{i}=\mathbf{Q}_{i, 1}, z_{i, 2}, \ldots, z_{i, r_{i}} \mathbf{\dagger} i=1,2, \ldots, n,
$$

respectively. Then a state vector $\vec{z}=\left(z_{1}, z_{2}, \ldots, z_{n}\right)$ of the system is element of

$$
\mathbf{Z}=\mathbf{Z}_{1} \times \mathbf{Z}_{2} \times \cdots \times \mathbf{Z}_{n}
$$

and the state space $\mathbf{Z}$ has $r_{1} \cdot r_{2} \cdots r_{n}$ elements. $\mathbf{Z}_{s}, \mathbf{Z}_{i}$ is called the frame of the indicator variables $z_{s}, z_{i} ; i=1,2, \ldots, n$; respectively. The structure function $z_{s}=\varphi(\vec{z})$ maps $\mathbf{Z}$ onto $\mathbf{Z}_{s}$. A multivalued coherent system with finite state sets $\mathbf{Z}_{s}, \mathbf{Z}_{i} ; i=1,2, \ldots, n$; and structure function $z_{s}=\varphi(\vec{z}), \quad \vec{z}=\left(z_{1}, z_{2}, \ldots, z_{n}\right) \in \mathbf{Z}$, is commonly defined as follows:

1) $\varphi$ is nondecreasing in each argument and

$$
\min _{i=1,2, \ldots, n} z_{i} \leq \varphi(\vec{z}) \leq \max _{i=1,2, \ldots, n} z_{i}
$$

Usually, the elements of the $\mathbf{Z}_{i}$ are real numbers. Otherwise, a total order in the set $\mathbf{Z}_{1} \cup \mathbf{Z}_{2} \cup \cdots \cup \mathbf{Z}_{n}$ must be given (see, for instance, [1, 11, 19]). In these papers it is generally assumed that the state spaces $\mathbf{Z}_{s}$ and $\mathbf{Z}_{i}$ are identical or $r_{s}=2$ and $r_{i}>2$. Multivalued systems with nondenumerable state spaces are, for instance, considered in [6]. Here a partial generalization of these models is dealt with: The state spaces $\mathbf{Z}_{s}, \mathbf{Z}_{1}, \mathbf{Z}_{2}, \ldots, \mathbf{Z}_{n}$ need not be identical (although this can be assumed without loss of generality) and the structure function $\varphi$ need not be nondecreasing. However, as in the previous section, the state $z_{s}$ of the system can only assume values 0 or 1 , i.e. $\mathbf{Z}_{S}=\{0,1\}$ (system is not available, is available). To be able to present an algorithm for constructing a disjoint sum form of the structure function, concepts introduced in section 2 have to be generalized. Let $Z \subseteq \mathbf{Z}_{i}$. A set constraint (SC) over $z_{i}$ with respect to $Z$ is denoted as $\left\langle z_{i} \in Z\right\rangle$ and defined by

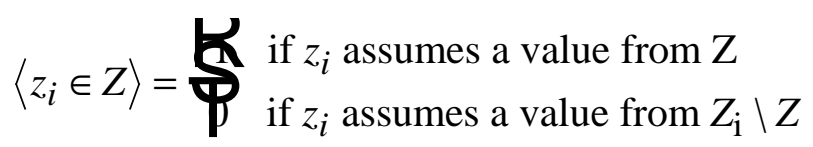

Thus, $Z$ is that subset of states, in which element $e_{i}$ operates satisfactorily. From the point of view of logic, $\left\langle z_{i} \in Z\right\rangle$ is a predicate, which is true iff $z_{i}$ assumes a value from $Z$. Obviously, 
if $\mathbf{Z}_{i}=\mid 0,1 \mathbf{C}$, then the Boolean variable $z_{i}$ is equivalent to $\left\langle z_{i} \in \mathbf{Z}_{i}\right\rangle$. Hence, SC's are generalizations of Boolean variables with

$$
0=\left\langle z_{i} \in \varnothing\right\rangle\left\langle\left\langle z_{i} \in \mathbf{Z}_{i}\right\rangle=1\right.
$$

Let $Z, Z^{\prime} \subseteq \mathbf{Z}_{i}, Z^{\prime \prime} \subseteq \mathbf{Z}_{j}$. Disjunction, conjunction and negation of SC's are defined as

Disjunction: $\left.\quad\left\langle z_{i} \in Z^{\prime}\right\rangle \vee\left\langle z_{j} \in Z^{\prime \prime}\right\rangle=\max \boldsymbol{\Theta}_{i} \in Z^{\prime}\right\rangle,\left\langle z_{j} \in Z^{\prime \prime}\right\rangle \mathbf{j}$

Conjunction: $\left.\quad\left\langle z_{i} \in Z^{\prime}\right\rangle \wedge\left\langle z_{j} \in Z^{\prime \prime}\right\rangle=\min \boldsymbol{\Theta}_{i} \in Z^{\prime}\right\rangle,\left\langle z_{j} \in Z^{\prime \prime}\right\rangle \mathbf{j}$

Negation: $\quad \overline{\left\langle z_{i} \in Z\right\rangle}=\left\langle z_{i} \in \mathbf{Z}_{i} \backslash Z\right\rangle$

In particular, for SC's over the same variable,

Disjunction $\quad\left\langle z_{i} \in Z^{\prime}\right\rangle \vee\left\langle z_{i} \in Z^{\prime \prime}\right\rangle=\left\langle z_{i} \in Z^{\prime} \cup Z^{\prime \prime}\right\rangle$

Conjunction $\quad\left\langle z_{i} \in Z^{\prime}\right\rangle \wedge\left\langle z_{i} \in Z^{\prime \prime}\right\rangle=\left\langle z_{i} \in Z^{\prime} \cap Z^{\prime \prime}\right\rangle$

Two SC's $\left\langle z_{i} \in Z^{\prime}\right\rangle$ and $\left\langle z_{j} \in Z^{\prime \prime}\right\rangle$ are said to be disjoint if $\left\langle z_{i} \in Z^{\prime}\right\rangle \wedge\left\langle z_{j} \in Z^{\prime \prime}\right\rangle=0$. If $i=j$, then $\left\langle z_{i} \in Z^{\prime}\right\rangle$ and $\left\langle z_{i} \in Z^{\prime \prime}\right\rangle$ being disjoint is equivalent to $Z^{\prime} \cap Z^{\prime \prime}=\varnothing$.

An SC $\left\langle z_{i} \in Z\right\rangle$ is called proper if $Z \neq \varnothing$ and $Z \neq \mathbf{Z}_{i}$.

An $S C$-clause (SC-term) is a disjunction (conjunction) of proper SC's with every variable $z_{i}$ occurring at most once.

For any two $(0,1)$-functions $f$ and $g$ defined on $\mathbf{Z}$,

$$
f \wedge g=\min (f, g) \text { and } f \vee g=\max (f, g)
$$

and $f=f(\vec{y})$ and $g=g(\vec{z})$ are called disjoint if $f(\vec{y}) \wedge g(\vec{z})=0$

for all $\vec{y}, \vec{z} \in \mathbf{Z}$. ( $f$ and $g$ may actually only depend on $k, k<n$, of the variables $z_{1}, z_{2}, \ldots, z_{n}$. In this case, the residual $n-k$ variables are irrelevant to $f$ and $g$ and can be deleted.) If $f$ and $g$ are disjoint, then

$$
f \vee g=f+g
$$

Let $Z_{i_{k}} \subset \mathbf{Z}_{i_{k}} ; k=1,2, \ldots, r ; 0<r<n$, and $f$ be the corresponding SC-term, i.e.

$$
f=\left\langle z_{i_{1}} \in Z_{i_{1}}\right\rangle \wedge\left\langle z_{i_{2}} \in Z_{i_{2}}\right\rangle \wedge \cdots \wedge\left\langle z_{i_{r}} \in Z_{i_{r}}\right\rangle
$$

As in chapter 2 , it will be assumed that the $z_{1}, z_{2}, \ldots, z_{n}$ are independent. Then, since SC's are random (0-1)-variables,

$$
\left.\left.\left.P(f=1)=E(f)=P \boldsymbol{Q}_{i_{1}} \in Z_{i_{1}}\right\rangle=1 \mathbf{j} P \boldsymbol{Q}_{i_{2}} \in Z_{i_{2}}\right\rangle=1 \mathbf{j} \cdots P \boldsymbol{Q}_{i_{r}} \in Z_{i_{r}}\right\rangle=1 \mathbf{j}
$$


with

$$
\left.P \boldsymbol{C}_{k} \in Z_{k}\right\rangle=1 \boldsymbol{7} \sum_{z_{k, j} \in Z_{k}} P\left(z_{k}=z_{k, j}\right)
$$

Let the structure function of a possibly noncoherent multivalued system be given in the form

$$
\varphi(\vec{z})=f_{1} \vee f_{2} \vee \cdots \vee f_{m},
$$

where the $f_{k}$ are proper terms with SC's over all or some of the $z_{1}, z_{2}, \ldots, z_{n}$. The usefulness of transforming structure function of type (5) into disjoint sum forms is motivated as in chapter 2. The following algorithm is an adaptation of algorithm 1 to noncoherent system functions with multi-valued arguments [3, 20]. It is based on a version firstly presented in [18]. An alternative approach using the information that every element is in exactly one mode is presented in [4].

\section{Algorithm 2}

; input: $\varphi=f_{1} \vee f_{2} \vee \cdots \vee f_{m}$ (order: $f_{j}$ contains not more SC's than $f_{j+1}$ )

; output: sets $M_{i, j} ; j=1, \ldots, r ; i=1, \ldots, j-1$

for $j=1$ to $m$

$$
\begin{aligned}
& M_{0, j}:=\bigcap_{j} \subseteq \\
& \text { for } i=1 \text { to } j-1 \\
& M_{i, j}:=\varnothing \\
& \text { for all } D \text { in } M_{i-1, j} \\
& \text { if } D \text { and } f_{i} \text { are disjoint, then add } D \text { to } M_{i, j}
\end{aligned}
$$

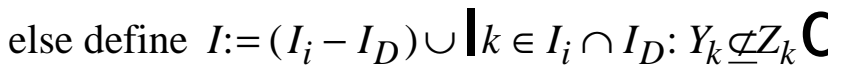

$$
\begin{aligned}
& \text { with } f_{i}=\underset{k \in I_{i}}{\wedge}\left\langle z_{k} \in Z_{k}\right\rangle \text { and } D=\underset{k \in I_{D}}{\wedge}\left\langle z_{k} \in Y_{k}\right\rangle \\
& \text { if } I=\left.\right|_{i_{1}, i_{2}, \ldots, i_{t}} \mathbf{Q} \varnothing \text {, then add the following formulas to } M_{i, j} \text { : } \\
& D \wedge\left\langle z_{i_{1}} \in \mathbf{Z}_{i_{1}} \backslash Z_{i_{1}}\right\rangle \\
& D \wedge\left\langle z_{i_{1}} \in \mathbf{Z}_{i_{1}}\right\rangle \wedge\left\langle z_{i_{2}} \in \mathbf{Z}_{i_{2}} \backslash Z_{i_{2}}\right\rangle \\
& D \wedge\left\langle z_{i_{1}} \in Z_{i_{1}}\right\rangle \wedge \cdots \wedge\left\langle z_{i_{t-1}} \in Z_{i_{t-1}}\right\rangle \wedge\left\langle z_{i_{t}} \in \mathbf{Z}_{i_{t}} \backslash Z_{i_{t}}\right\rangle
\end{aligned}
$$


Note that one has to make sure that the algorithm generates only proper SC-terms. By using an appropriate data structure for representing SC-terms, this can be done efficiently. The sets $M_{i, j}$ only contain SC-terms and the corresponding disjoint sum form is

$$
\varphi=\sum_{j=1}^{m} \sum_{D \in M_{j-1, j}} D
$$

Example 2 Consider variables $z_{1}, z_{2}, z_{3}$ with identical frame $\mathbf{Z}=\mid \mathbf{1 , 2 , 3 , 4} \mathbf{C}$. Let us assume a non-monotone system function of type (5) given by $\varphi=f_{1} \vee f_{2}$ with

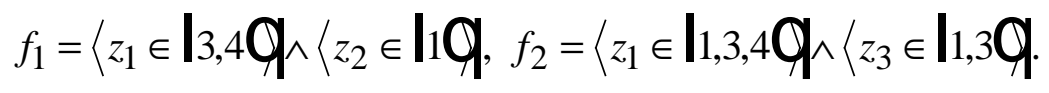

Applying algorithm 2 yields:

$j=1:$ Initiate $M_{0,1}:=\mid f_{1} \mathbf{C}$

$j=2:$ Initiate $M_{0,2}:=\mathbf{I}_{2} \mathbf{C}$

$i=1$ : The only element of $M_{0,2}$ is not disjoint with $f_{1}$. Hence,

determine $I=\mid 1,2 C$ and construct formulas

$$
\begin{aligned}
& \left\langlez _ { 1 } \in | 1 , 3 , 4 \mathbf { Q } | \wedge \left\langle z_{3} \in|1,3 \mathbf{Q}| \wedge \overline{\left\langle z_{1} \in|3,4 \mathbf{Q}|\right.}\right.\right.
\end{aligned}
$$

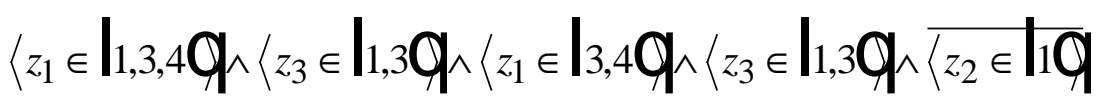

Simplifying these formulas to obtain SC-terms yields

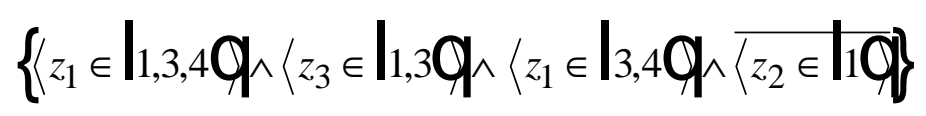

Hence, the system availability becomes

$$
\begin{aligned}
& P(\varphi=1)=\sum_{D \in M_{0,1}} P(D=1)+\sum_{D \in M_{1,2}} P(D=1)
\end{aligned}
$$

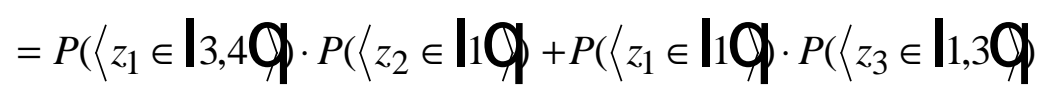

$$
\begin{aligned}
& +P\left(\langle z _ { 1 } \in \mathbf { | } 3 , 4 \mathbf { Q } | \cdot P \left(\langle z _ { 2 } \in \mathbf { | } 2 , 3 , 4 \mathbf { Q } | \cdot P \left(\left\langlez_{3} \in \mathbf{|}, 3 \mathbf{Q}|\cdot|\right.\right.\right.\right.
\end{aligned}
$$

\section{REFERENCES}

[1] A.M. ABOUAMMOH,. and M.A. Al-KADI, Multistate coherent systems of order $k$, Microelectronics and Reliability, 35, 1415-1421 (1995).

[2] J.A. ABRAHAM, An improved algorithm for network reliability, IEEE Trans. on Reliab., 28, 58-61 (1979). 
[3] B.A. ANRIG, A generalization of the algorithm of Abraham, In: M. Nikulin, M. and Limios, N. (eds.), Proc. of the $2^{\text {nd }}$ Int. Conf. on Mathematical Methods in Reliability, II, Bordeaux, July 3-4, 2000, 95-98 (2000).

[4] B. ANRIG, and P.A. MONNEY, Using propositional logic to compute the probability of diagnoses in multistate systems, Internaional. Journal of Approximate Reasoning 20, 113-143 (1999).

[5] M.O. BALL, and J.S. PROVAN, Disjoint products and efficient computation of reliability, Operations Research, 36, 703-715 (1988).

[6] L.A. BAXTER, Continuum structures I, Journal of Applied Probabillity, 21, 802-815 (1984).

[7] F. BEICHELT, Reliability and Maintenance Theory (in German), Teubner, Stuttgart (1993).

[8] F. BEICHELT and L. SPROß, An improved Abraham-method for generating disjoint sums, IEEE Transactions on Reliability, 36, 70-74 (1987).

[9] F. BEICHELT and L. SPROß, Comment on "An improved Abraham-method for generating disjoint sums.”, IEEE Transactions on Reliability, 38, 422-424 (1989).

[10] R. BERTSCHY and P.A. MONNEY, A generalization of the algorithm of Heidtmann to non-monotone formulas. Journal of Computational and Applied Mathematics, 76, 55-76 (1996).

[11] W.S. GRIFFITH, Multistate reliability models, Journal of Applied Probability, 17, 735-744 (1980).

[12] R. HAENNI, R. and LEHMANN, Building argumentation systems on set constraint logic, In: Bouchon-Meunier, B. et al (eds.), Information, Uncertainty and Fusion, Kluwer Academic Publishers, 393-406 (2000).

[13] R. HÄHNLE, Advanced many-valued logics, In: Gabbey, D. (ed.), Handbook of Philosoph. Logic, Vol. 6: Alternatives to Classical Logic I (2. ed.), Kluwer, 2000.

[14] K.D. HEIDTMANN, Smaller sums of disjoint products by subproduct inversion, IEEE Transactions on Reliability, 38, 305-311 (1989).

[15] J. KOHLAS, Reliability and Availability (in German). Teubner, Stuttgart (1987).

[16] J. KOHLAS, B. ANRIG, R. HAENNI,. and P.A. MONNEY, Model-based diagnostics and probabilistic assumption-based reasoning, Artificial Intelligence, 104, 71-106, (1998).

[17] J. KOHLAS, R. HAENNI. and N. LEHMANN, Probabilistic argumentation systems, In: Kohlas, J. and Moral, S. (eds.), Handbook of Defeasible Reasoning and 
http://orion.journals.ac.za/

Uncertainty Management Systems, vol. 5: Algorithms for Uncertainty andDefeasible Reasoning, Kluwer (2000).

[18] J. KOHLAS,. and P.A. MONNEY, A Mathematical Theory of Hints, An Approach to the Dempster-Shafer Theory of Evidence, Vol. 25 of Lecture Notes in Economics and Mathematical Systems, Springer (1995).

[19] F.C. MENG, Characterizing the Barlow-Wu structure functions, Naval Research Logistics, 41, 661-668 (1994).

[20] P.A. MONNEY,. and B. ANRIG, Computing the probability of formulas representing events in product spaces, In: Bouchon-Meunier, B. et al (eds.), Information, Uncertainty and Fusion, Kluwer Academic Publishers, 197-208 (2000).

[21] S. RAI, M. VEERARAGHAVAN, M. and S. TRIVEDI, A survey of efficient reliability computation using disjoint products approach, Networks, 25, 147-163 (1995).

[22] J. TORREY, A pruned tree approach to reliability computation, IEEE Transactions on Reliability, 32, 170-174 (1983).

[23] M. VEERARAGHAVAN,. and S. TRIVEDI, Multiple variable inversion techniques, Chapter 2 in: Misra, K. (ed.), New trends in system reliability evaluation, Elsevier, 3974, (1994). 